Article

\title{
Immobilization of Tyrosinase from Avocado Crude Extract in Polypyrrole Films for Inhibitive Detection of Benzoic Acid
}

\author{
André Brisolari ${ }^{\dagger}$ and Débora Gonçalves * \\ Instituto de Física de São Carlos, Universidade de São Paulo, Av. Trabalhador São-Carlense 400, \\ CP 369, 13560-970, São Carlos - SP, Brazil \\ $\dagger$ In memoriam. \\ * Author to whom correspondence should be addressed; E-Mail: gdebora@if.sc.usp.br; \\ Tel.: +55-16-3373-9825; Fax: +55-15-3371-5365.
}

Received: 14 March 2014; in revised form: 11 June 2014 / Accepted: 27 June 2014 /

Published: 3 July 2014

\begin{abstract}
Inhibition-based biosensors were developed by immobilizing tyrosinase (Tyr, polyphenol oxidase) from the crude extract of avocado fruit on electrochemically prepared polypyrrole (PPy) films. The biosensors were prepared during the electropolymerization of pyrrole in a solution containing a fixed volume of the crude extract of avocado. The dependence of the biosensor responses on the volume used from the crude extract, values of $\mathrm{pH}$ and temperature was studied, and a substrate, catechol, at different concentrations, was amperometrically detected by these biosensors. Benzoic acid, a competitive inhibitor of Try, was added to the catechol solutions at specific concentrations aimed at obtaining the inhibition constant, $\mathrm{K}_{\mathrm{m}}$, which ranged from 1.7 to $4.6 \mathrm{mmol} \cdot \mathrm{L}^{-1}$ for 0.0 and $60 \mu \mathrm{mol} \cdot \mathrm{L}^{-1}$ of benzoic acid, respectively. Studies on the inhibition caused by benzoic acid by using PPy/Try films, and catechol as a substrate, allowed us propose how to develop, under optimized conditions, simple and low-cost biosensors based on the use of avocado fruit.
\end{abstract}

Keywords: inhibition; polypyrrole; biosensors; food preservatives; polyphenol oxidase

\section{Introduction}

Biosensors based on the immobilization of enzymes within polymer matrices are considered to be a promising technology for detection of species of interest [1]. Among several examples of biosensors, 
one particular category is capable of analyzing how the concentration of an analyte varies in the presence of an enzyme inhibitor, for a determined period of time, i.e., the so-called inhibition-based biosensors [2]. Enzymatic inhibition biosensors have been applied to the analysis of pesticides, heavy-metals, and preservatives in foods [3,4]. The mechanisms of enzyme inhibition are often complex, and can be studied by exposing an immobilized enzyme to a specific inhibitor and, therefore, interfering with the active sites of the enzyme to either retard or accelerate reaction rates. These mechanisms can be classified into different types: reversible, irreversible or mixed, and the main difference between them is that irreversible enzyme-inhibitor interactions yield hydrolytic and oxidative destruction of the enzyme [3].

Benzoic acid has been used as a preservative in a variety of food products and beverages in order to protect them against deterioration due to antimicrobial properties [5,6]. However, benzoic acid at levels higher than those permitted by legislation may be potentially harmful to human health. Benzoic acid in foodstuffs has been determined by methods, such as high performance liquid chromatography, thin layer chromatography, and gas chromatography, which are expensive, time-consuming, and demand qualified technicians [7]. As an alternative, amperometric biosensors have been reported in the monitoring of benzoic acid due to such advantages as effectiveness, simplicity, and low cost $[8,9]$. For inhibition-based biosensors, the immobilization step is of utmost importance, and tyrosinase (Tyr, polyphenol oxidase) has been chosen for the design of biosensors based on the presence of benzoic acid as a competitive, reversible enzyme inhibitor [8-11].

Tyr has been found in nature from sources such as vegetables, fruits, and mushrooms. It contains a binuclear, active copper site responsible for catalyzing the hydroxylation of monophenols to $o$-diphenols, and subsequent oxidation to $o$-quinones, which can be reduced back to $o$-diphenols [12]. Tyr also contains high-affinity binding sites for aromatic compounds, which justifies its use in inhibition-based biosensors, not only for quantification but, also, in studies focused on effects of pigmentation [13].

Inhibitor biosensors based on the immobilization of Tyr on polyaniline [10,11], graphite-Tefon composites [14], and copolymers [15] have been studied for the determination of benzoic acid; however, in all cases, Tyr was commercially obtained. Here, the novelty of our studies lies in the use of a stable, biocompatible polymer film, polypyrrole (PPy), as a matrix for immobilizing Tyr under mild oxidative conditions, in aqueous solutions.

Our interest was to obtain the optimum working conditions when the biosensors were prepared using stabilized, active Tyr in its natural source, avocado fruit. A systematic evaluation of the inhibition effects of PPy/Tyr films in the presence of benzoic acid has indicated that optimized conditions yield efficient, simple, and low-cost biosensors by using crude extract of avocado fruit as an enzymatic source.

\section{Experimental Section}

Pyrrole was purified by vacuum distillation prior to be used. Tyrosinase (Tyr, polyphenol oxidase) was used as crude extract of avocado fruit (Persea americana, bought at local food markets) according to the procedures described in the literature [16]. 
The avocado pulp (25 g) was homogenized in a blender for 3 min with $100 \mathrm{~mL}$ of phosphate buffer ( $\mathrm{pH}$ 7.0) and $2.5 \mathrm{~g}$ of Super Polyclar $\mathrm{R}$, which is an insoluble polymer capable of binding to polyphenols, inhibiting browning, and producing stable enzymatic crude extracts over several months. This procedure has yielded an homogeneous solution, which was filtered by using four layers of cheesecloth, centrifuged at $15,000 \mathrm{rpm}$ for $30 \mathrm{~min}$ at $4{ }^{\circ} \mathrm{C}$, and then, stored in a fridge.

The activity of Tyr extracted from avocado tissues was assayed in triplicate with a U-2900 Hitachi spectrophotomer in the UV-VIS range using $2.8 \mathrm{~mL}$ of $0.1 \mathrm{~mol} \cdot \mathrm{L}^{-1}$ catechol in $0.1 \mathrm{~mol} \cdot \mathrm{L}^{-1}$ phosphate buffer at $\mathrm{pH} 7.0$ and $0.2 \mathrm{~mL}$ of the crude extract of avocado. The maximum absorbance was measured at $410 \mathrm{~nm}$, owing to the formation of $o$-quinones, and $15 \mathrm{~min}$ after starting the enzymatic reaction, allowing one to obtain the activity of Tyr as crude extract of avocado. One unit of enzyme activity can be defined as the amount of enzyme that causes an increase of 0.001 units of absorbance per min. The activity of Tyr using catechol as substrate was obtained as $6923 \pm 437 \mathrm{U} \cdot \mathrm{mL}^{-1}$.

The immobilization of Tyr was carried out during the electrosynthesis of the PPy films by adding $100 \mu \mathrm{L}$ of the crude extract of avocado in the electropolymerization medium containing $0.1 \mathrm{~mol} \cdot \mathrm{L}^{-1}$ $\mathrm{LiClO}_{4}$ and $0.07 \mathrm{~mol} \cdot \mathrm{L}^{-1}$ pyrrole.

A potentiostat/galvanostat PAR Versastat II was used for the electrochemical measurements. The electropolymerization of pyrrole was performed by cyclic voltammetry at $50 \mathrm{mV} \cdot \mathrm{s}^{-1}$ between $0.0 \mathrm{~V}$ and $1.0 \mathrm{~V}$ vs. SCE (saturated calomel electrode) in an aqueous medium containing $0.1 \mathrm{~mol} \cdot \mathrm{L}^{-1} \mathrm{LiClO}_{4}$ and $0.07 \mathrm{~mol} \cdot \mathrm{L}^{-1}$ pyrrole up to reach 10 cycles in a glass cell containing the reference electrode, a $\mathrm{Pt}$ plate as the counter electrode, a $\mathrm{Pt}$ rod as the working electrode, and nitrogen purging. Chronoamperometry was used in order to detect catechol and benzoic acid at a fixed potential of $240 \mathrm{mV}$ for $0.2 \mathrm{~s}$ in phosphate buffer at $\mathrm{pH} 7.0$.

\section{Results and Discussion}

Initially, we studied how to obtain optimized PPy/Tyr biosensors by using crude extract of avocado and a set of defined parameters: operational potential, amount of crude extract of avocado, $\mathrm{pH}$, and temperature. The immobilization procedure relied on the entrapment of Tyr within the PPy matrix in an aqueous medium containing $0.1 \mathrm{~mol} \cdot \mathrm{L}^{-1} \mathrm{LiClO}_{4}, 0.07 \mathrm{~mol} \cdot \mathrm{L}^{-1}$ pyrrole, and crude extract of avocado at a fixed volume $(100 \mu \mathrm{L})$.

Uniform PPy/Tyr films were obtained by cyclic voltammetry after ten complete cycles by applying a final potential of $1.0 \mathrm{~V}$, which proved to be high enough to promote a controllable oxidation of pyrrole, but low enough to avoid degradation (or electroinactivity) of the polymer matrix.

The voltammograms obtained during the electropolymerization of pyrrole without and with Tyr (crude extract of avocado, $100 \mu \mathrm{L}$ ), from $-1.0 \mathrm{~V}$ to $1.0 \mathrm{~V}$ at $50 \mathrm{mV} \cdot \mathrm{s}^{-1}$ in $0.1 \mathrm{~mol} \cdot \mathrm{L}^{-1} \mathrm{LiClO}_{4}$ and $0.07 \mathrm{~mol} \cdot \mathrm{L}^{-1}$ pyrrole, are essentially the same (Figure 1 ). There are only slight differences in these curves, giving an indication that Tyr has no significant influence on the electrochemical response of the electrode surface. The most visible difference in these curves is that pyrrole oxidizes at more positive potentials when in the presence of Tyr (see arrows in Figure 1).

The total charges after completing ten cycles were practically the same for both processes, but the presence of Tyr yields films with a different morphology, as can be seen in Figure 2. 
Figure 1. Cyclic voltammograms during the preparation of the PPy (a) and PPy/Try (b) films in $0.1 \mathrm{~mol} \cdot \mathrm{L}^{-1} \mathrm{LiClO}_{4}, 0.07 \mathrm{~mol} \cdot \mathrm{L}^{-1}$ pyrrole, without (a), and with (b), crude extract of avocado at a fixed volume $(100 \mu \mathrm{L})$.
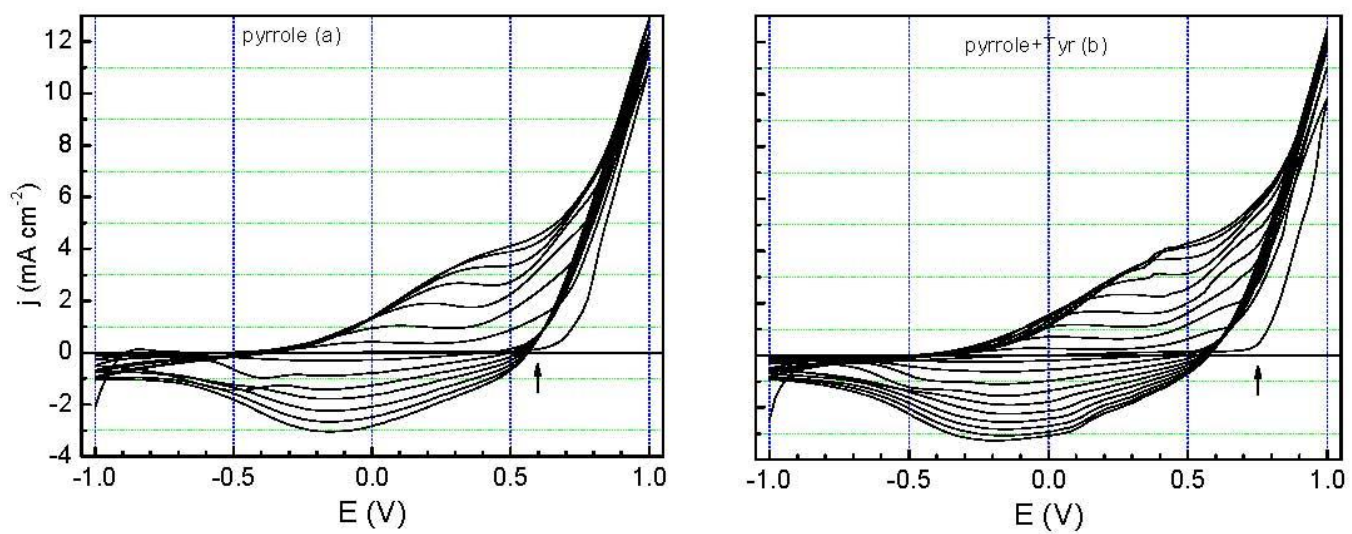

Figure 2. Scanning electron microscope (SEM) images for the (a) PPy and (b) PPy/Try films obtained after 10 voltammetric cycles.
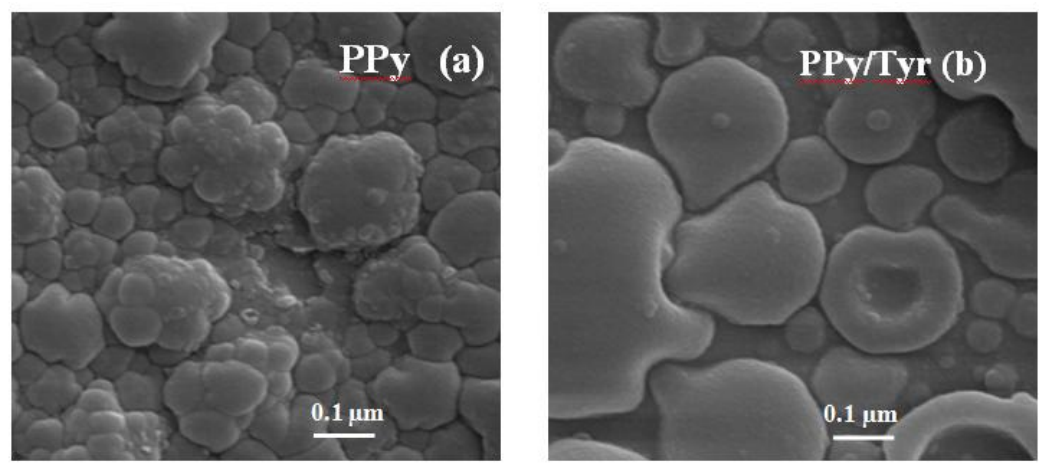

The morphology of PPy films is typically cauliflower-like [17], with globular structures covering the entire surface of the film (Figure 2a). The presence of Tyr in the electropolymerization medium has yielded a more compact structure, and although those films have been produced with practically the same values of polymerization charge, they are morphologically different. It has been addressed in the literature that when electrochemically immobilized, Tyr becomes entrapped in the polymer matrix (poly-3,4-ethylenedioxythiophene, PEDOT [18], and a copolymer [15]) as also expected here by using crude extract of avocado as a source of Tyr.

The FTIR spectra of the films show the typical bands of a predominant polymer matrix, PPy; but, for the PPy/Tyr film, a shift of the main band positions to higher wavenumbers (blue shifted) is evident as addressed in Figure 3. Similar effect has been reported by us after immobilizing phytase in PPy films [19]. Such shifts of the main bands in the FTIR spectra of PPy are indicative of an electrostatic interaction between Tyr and its matrix, PPy, due to differences in charge signals. Besides, the peak at ca. $1700 \mathrm{~cm}^{-1}$, which has been assigned to the overoxidation of PPy by the presence of carbonyl groups at the $\beta$-position of the pyrrole ring [20], is not found in the spectrum of the PPy-Tyr film. 
Figure 3. FTIR spectra for the PPy (a) and PPy/Try (b) films obtained after 10 voltammetric cycles.

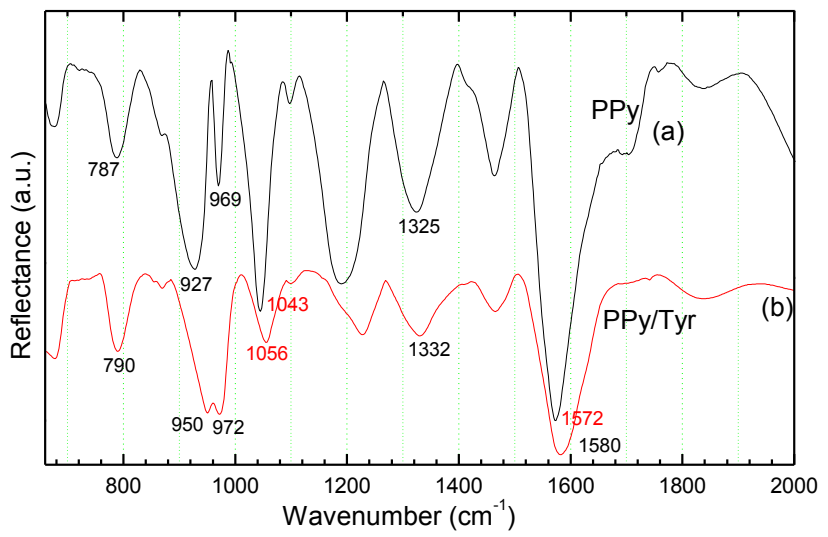

The voltammetric responses of the PPy and PPy/Tyr films without and with a fixed concentration of catechol, $1.0 \times 10^{-3} \mathrm{~mol} \cdot \mathrm{L}^{-1}$, have evidenced remarkable differences in their voltammetric responses (Figure 4). From Figure 4A, the operational potential was defined as $+240 \mathrm{mV}$ for all subsequent experiments. The PPy film did not show selectivity for catechol, and its voltammetric response was used as the calibration curve. When Tyr is immobilized in the PPy matrix, the film shows a different voltammetric response, which can be related to a different morphology, thus a different mobility of ions into the film. These differences seen in the voltammetric curves of the PPy films are quite common in films of different morphology, such as PPy [21], and poly-3-alhylthiophenes [22].

When added at different concentrations in a medium containing a substrate (catechol), benzoic acid clearly affects the voltammetric responses of the PPy/Tyr film due to an inhibitory effect, as it can be verified in Figure 4B. The presence of benzoic acid results on a decrease in the peak currents (see arrows in Figure 4B), which are related to enzymatic reactions involving $o$-quinones.

Figure 4. Cyclic voltammograms for: (A) PPy (a) and PPy/Try (b) films in $0.1 \mathrm{~mol} \cdot \mathrm{L}^{-1}$ $\mathrm{LiClO}_{4}$ without and with $1,0 \mathrm{mmol} \cdot \mathrm{L}^{-1}$ catechol (PPy/Try/catechol) (c); (B) PPy/Try films in $0.1 \mathrm{~mol} \cdot \mathrm{L}^{-1}$ phosphate buffer (a) and $50 \mu \mathrm{L}$ of catechol without (b) and with $100 \mu \mathrm{L}(\mathbf{c})$; $200 \mu \mathrm{L}$ (d) $300 \mu \mathrm{L}$ (e) of benzoic acid; $50 \mathrm{mV} \cdot \mathrm{s}^{-1}$.

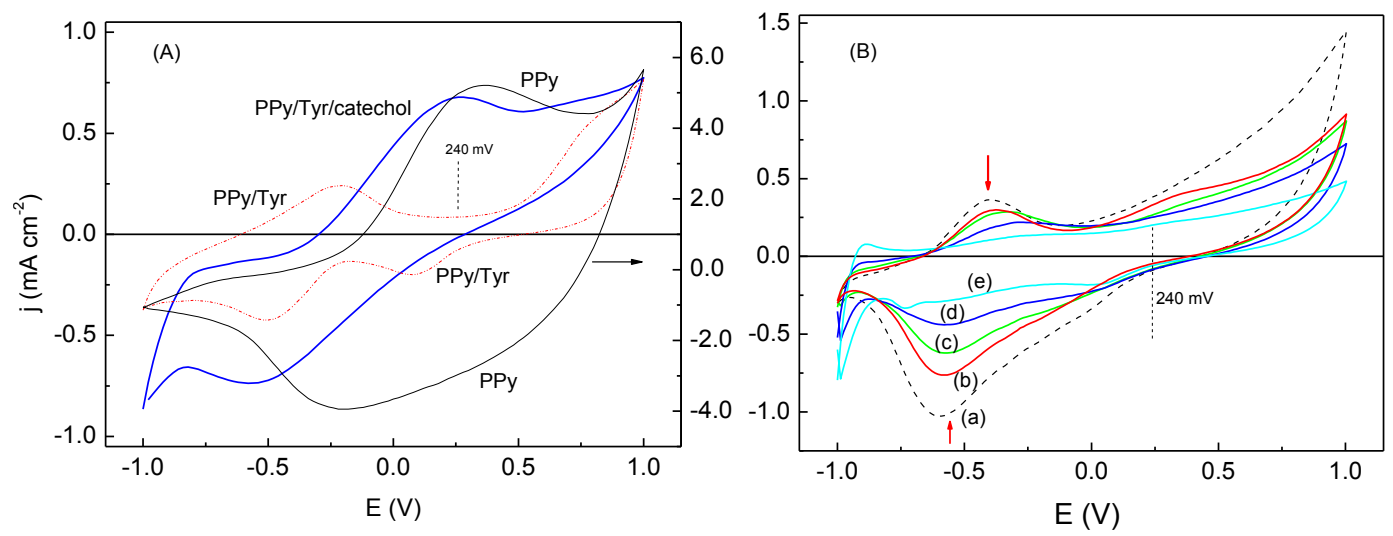

For the preparation of the PPy/Tyr films, aliquots from $20 \mu \mathrm{L}$ to $140 \mu \mathrm{L}$ of the crude extract of avocado were added sequentially to a solution containing $0.07 \mathrm{~mol} \cdot \mathrm{L}^{-1}$ pyrrole, $0.1 \mathrm{~mol} \cdot \mathrm{L}^{-1}$ phosphate 
buffer, and $1.0 \times 10^{-3} \mathrm{~mol} \cdot \mathrm{L}^{-1}$ catechol. Thus, the steady-state currents of the working electrode $(\mathrm{Pt})$ in these solutions were obtained at a fixed potential, $+240 \mathrm{mV}$, for each volume of the crude extract of avocado (Figure 5a). From these results, the optimal volume of the crude extract of avocado for obtaining the PPy/Tyr films was defined as $100 \mu \mathrm{L}$ since it yielded the highest response of current. Tyr has demonstrated a high specificity for phenolic compounds, in particular catechol, without considerable effects of interference at a low applied potential. Previous results have shown that common interfering substances in foodstuffs are lactic acid, sorbic acid, caffeine, and saccharin sodium [8,9], with benzoic acid effectively acting against bacterial development [6]. In this case, these interference substances did not show a considerable influence on the response of Tyr-based sensors towards benzoic acid. Only sorbic acid, which is a preservative against yeasts and moulds, yields a higher, but still low, signal increase, about 10\% [8]. For the PPy/Tyr films, we are expecting similar results, but further studies are necessary in order to confirm this issue.

The current densities were also examined after changing the $\mathrm{pH}$ from 5.0 to 8.5 in phosphate buffer, but keeping constant the volume of the crude extract of avocado, $100 \mu \mathrm{L}$, and concentration of catechol, $1.0 \times 10^{-3} \mathrm{~mol} \cdot \mathrm{L}^{-1}$ (Figure $5 \mathrm{~b}-\mathrm{A}$ ), and a maximum response has been achieved at $\mathrm{pH} 6.5$. In the presence of an inhibitor, benzoic acid, which was added separately to the solution at a fixed concentration, $1.0 \times 10^{-3} \mathrm{~mol} \cdot \mathrm{L}^{-1}$, a similar feature can be noticed in the current density $v s . \mathrm{pH}$ plot, but with lower current densities (Figure 5b-B). This type of response, i.e., lower currents in the presence of benzoic acid, has also been noted for polyaniline-polyacrylonitrile/Tyr [10] and polyaniline/Tyr [11] films. The optimum value of $\mathrm{pH}$ obtained here is in accordance to those reported in the literature, which ranged from 6.5 to $7.0[10,11]$.

Figure 5. Responses of current for PPy/Tyr films prepared in pyrrole, phosphate buffer, and catechol at different: (a) volumes of crude extract of avocado; (b) pHs without (A) and with (B) benzoic acid.
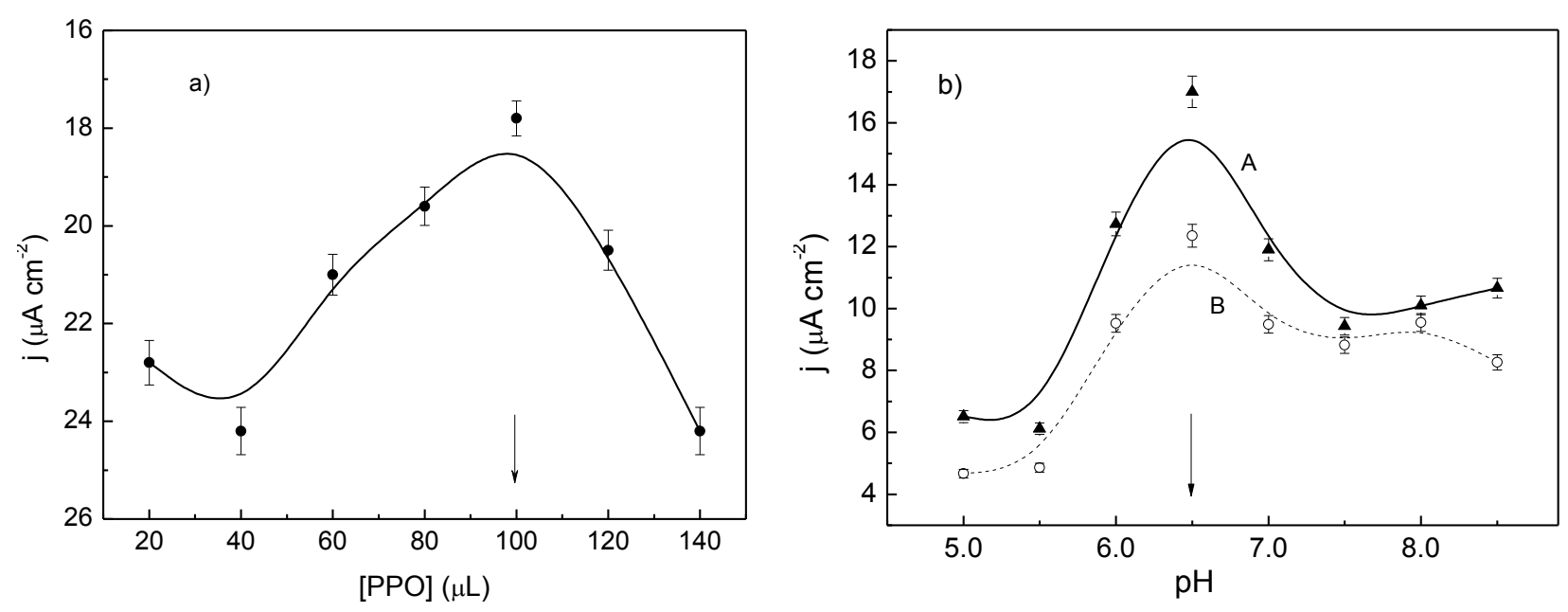

The current density also varies at different temperatures without and with benzoic acid (Figure 6). These results allowed us to obtain the optimum temperature for the PPy/Tyr biosensor with respect to the presence of catechol, $50-55{ }^{\circ} \mathrm{C}$, regardless of the presence of benzoic acid in solution. Besides, there is a clear indication that the inhibitor has no significant influence on the thermal stability of Tyr, 
at least after the immobilization within the PPy matrix. The maximum activity for either free Tyr or after being immobilized in polyaniline films was obtained at about $40{ }^{\circ} \mathrm{C}[10,11]$.

Figure 6. Responses of current for PPy/Tyr films prepared in pyrrole, phosphate buffer, and catechol at different temperatures without (A) and with (B) benzoic acid.

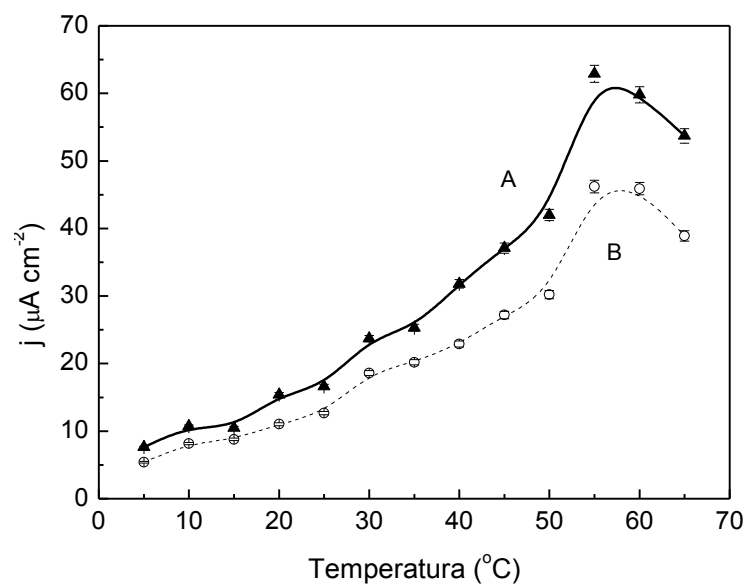

The current densities were also obtained at a fixed potential $(+240 \mathrm{mV})$ after varying the concentration of catechol, from $0.2 \times 10^{-3} \mathrm{~mol} \cdot \mathrm{L}^{-1}$ to $10 \times 10^{-3} \mathrm{~mol} \cdot \mathrm{L}^{-1}$, and benzoic acid, from 0 to $60 \mu \mathrm{mol} \cdot \mathrm{L}^{-1}$ (Figure 7a). A stable plateau was reached at about $6.0 \times 10^{-3} \mathrm{~mol} \cdot \mathrm{L}^{-1}$ of catechol, and a linear range reached up to a concentration of catechol of $4.0 \times 10^{-3} \mathrm{~mol} \cdot \mathrm{L}^{-1}$. The lowest concentration of catechol which had an influence on the current response of the biosensor was $0.5 \times 10^{-3} \mathrm{~mol} \cdot \mathrm{L}^{-1}$. The double-reciprocal Lineweaver-Burk plot was obtained without and with the presence of benzoic acid as shown in Figure 7b. Higher concentrations of benzoic acid implicated on an increase in the slope of the curves, thus indicating a competitive, inhibitory process caused by chelation of $\mathrm{Cu}$ atoms within the active site of Tyr [23].

Figure 7. (a) Current response and (b) Lineweaver-Burk plot vs. concentration of catechol for PPy/Tyr films at different concentrations of benzoic acid.
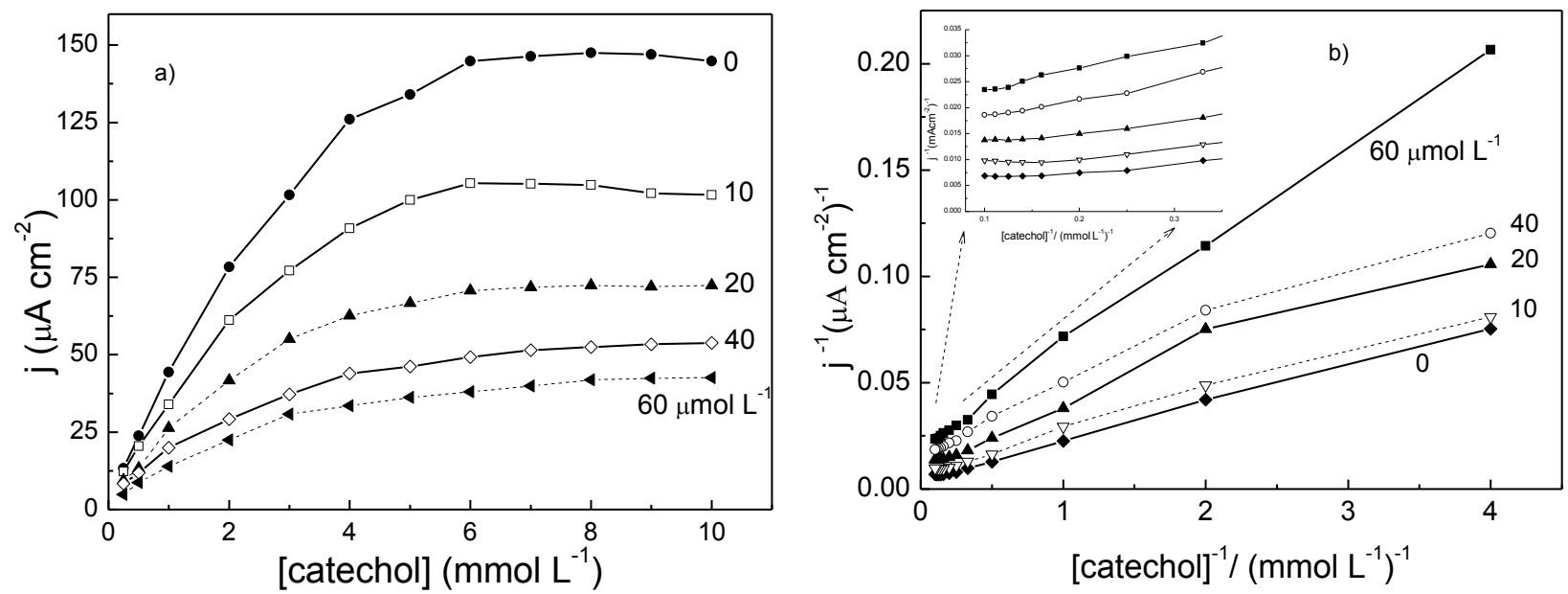
The values of apparent Michaelis-Menten constant, $\mathrm{K}_{\mathrm{m}}$, were calculated from the slopes of Figure $7 b$, evidencing that $K_{m}$ increases with the concentration of benzoic acid. When the concentration of the inhibitor varies with the concentration of catechol, the plot is named Dixon plot [24], which gives the values of inhibition constant, $\mathrm{K}^{\prime}{ }_{\mathrm{m}}$, for benzoic acid bonded to PPO, Figure 8 .

Figure 8. Dixon plot with the values of the inhibition constant, $\mathrm{K}_{\mathrm{m}}$, as indicated in the figure.

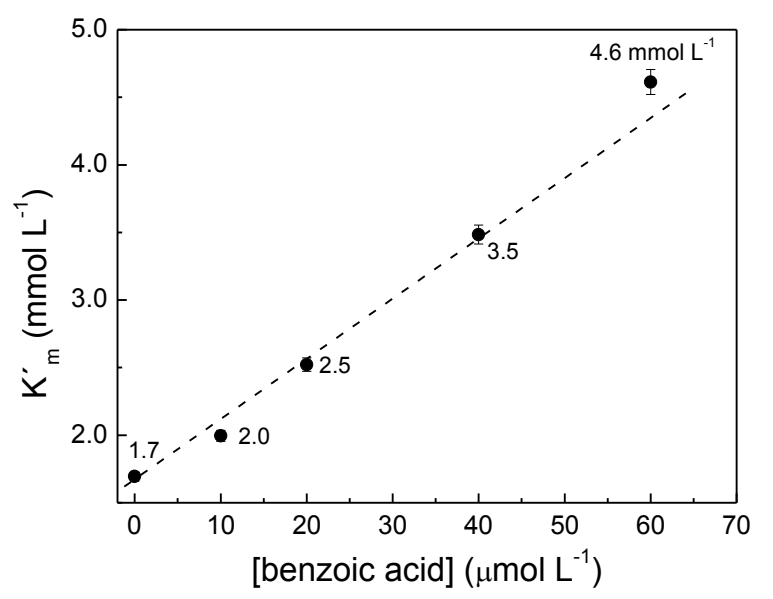

Figure 9a shows the current density as a function of time for a PPy/Tyr film at a fixed concentration of catechol, $2.0 \mathrm{mmol} \cdot \mathrm{L}^{-1}$, and after adding continuously aliquots of benzoic acid. Since the PPy/Tyr films show a sensibility to the presence of benzoic acid, two different concentrations of catechol were chosen upon varying the concentration of benzoid acid. Then, the inhibition percentages were calculated comparatively (Figure $9 \mathrm{~b}$ ) from the responses of the density current before and after adding benzoic acid, with a maximum inhibition percentage of about $80 \%$ after $50 \mu \mathrm{mol} \cdot \mathrm{L}^{-1}$.

The expression used for calculation of the inhibition percentages was $\mathrm{I}=$ degree of inhibition, current responses obtained without $\left(\mathrm{I}_{1}\right)$ and with $\left(\mathrm{I}_{2}\right)$ benzoic acid: $\mathrm{I}(\%)=100\left(\mathrm{I}_{1}-\mathrm{I}_{2}\right) / \mathrm{I}_{1}$.

Figure 9. Current responses for PPy/Tyr films at different concentrations of benzoic acid (a); (b) inhibition percentage vs. concentration of benzoic acid in the presence of catechol at $30 \mathrm{mmol} \cdot \mathrm{L}^{-1}$ and $20 \mathrm{mmol} \cdot \mathrm{L}^{-1}$.
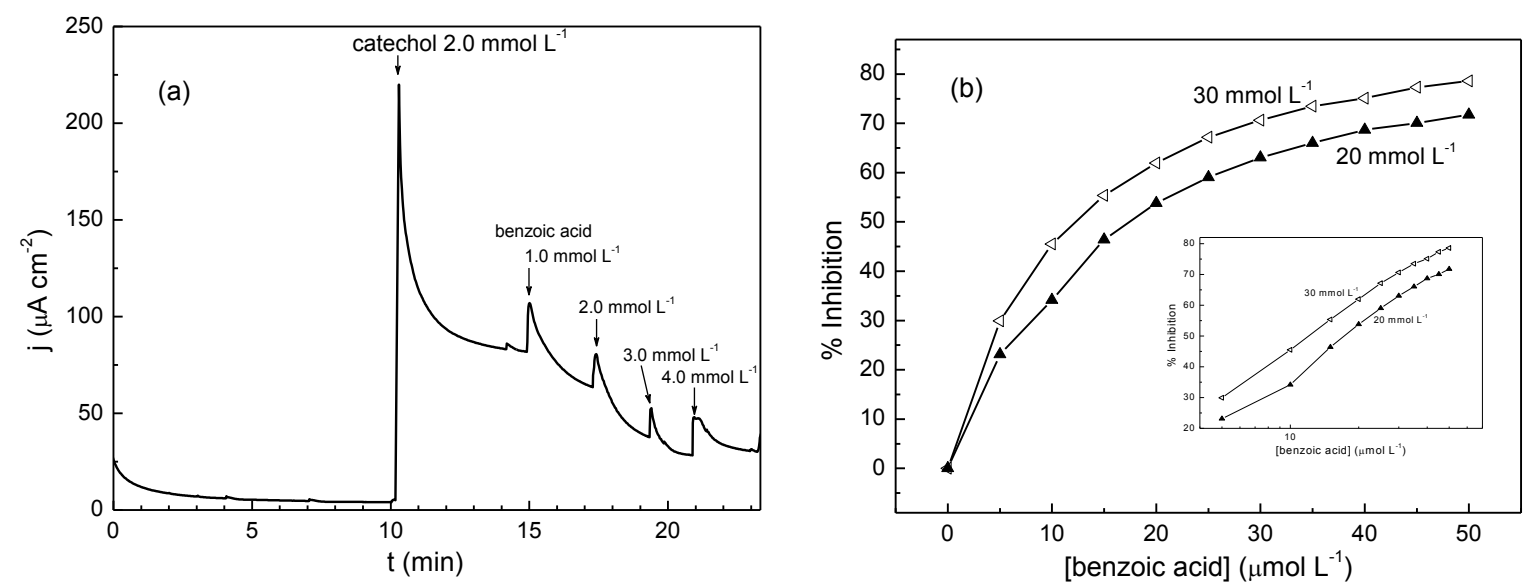
The degree of inhibition by benzoic acid for the PPy/Tyr film was found to be $39 \%$, in accordance with what was reported for different matrices using commercial Tyr [10,11]. When these results are represented by a linear plot (insert, Figure $9 \mathrm{~b}$ ), it is evident a variation of $60 \%$ for the current response at $50 \mu \mathrm{mol} \cdot \mathrm{L}^{-1}$ of benzoic acid added to the solution containing catechol.

For higher concentrations of catechol, the inhibition effect of a competitive inhibitor of Tyr such as benzoic acid does not have a significant effect [9]. The operational stability of the PPy/Tyr films was also examined in terms of the number of repetitive essays performed after 21 successive measurements (Figure 10).

Figure 10. Operational stability for PPy/Tyr films.

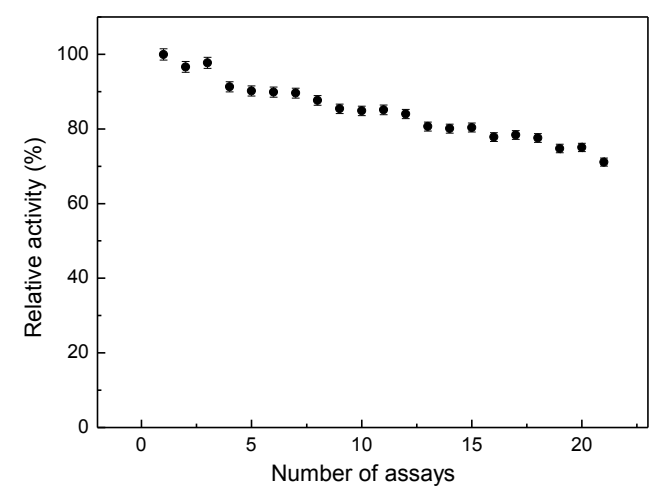

The activity of the PPy/Tyr films was successively measured, with Tyr maintaining 70\% of its initial activity after 21 days. This result is higher than those of commercial Tyr immobilized on similar polymeric matrices [17], thus indicating to be PPy/Tyr (crude extract of avocado) a reasonable alternative for detecting phenol and preservatives such as benzoic acid.

\section{Conclusions}

The possibility of using cheap Tyr extracted from avocado fruit pulp and immobilized in PPy films is a reality for the detection of phenolic compounds, such as catechol and benzoic acid as the enzymatic inhibitor. The entrapment of Tyr within the PPy matrix proved to be a simple and fast method, in particular in aqueous solution (without the use of organic solvents). The PPy/Tyr films exhibited a good performance in terms of detection range and response as compared to conventional biosensors built from commercial enzymes. Possible applications of this amperometric biosensor include the analysis of preservatives in food, and for environmental and industrial monitoring.

\section{Acknowledgments}

Fundação de Amparo à Pesquisa do Estado de São Paulo, FAPESP, and Conselho Nacional de Desenvolvimento Científico e Tecnológico (CNPq).

\section{Conflicts of Interest}

There is no conflict of interest. 


\section{References}

1. Lange, U.; Roznyatovskaya, N.V.; Mirsky, V.M. Conducting polymers in chemical sensors and arrays. Anal. Chim. Acta 2008, 614, 1-26.

2. Upadhyay, L.S.B.; Verma, N. Enzyme inhibition based biosensors: A review. Anal. Lett. 2013, 46, 225-245.

3. Amine, A.; Mohammadi, H.; Bourais, I.; Palleschi, G. Enzyme inhibition-based biosensors for food safety and environmental monitoring. Biosens. Bioelectron. 2006, 21, 1405-1423.

4. Arduini, F.; Amine, A.; Moscone, D.; Palleschi, G. Reversible enzyme inhibition-based biosensors: Applications and analytical improvement through diagnostic inhibition. Anal. Lett. 2009, 42, 1258-1293.

5. Tfouni, S.A.V.; Toledo, M.C.F. Determination of benzoic and sorbic acids in Brazilian food. Food Control 2002, 13, 117-123.

6. Montaño, A.; Sánchez, A.H.; Rejano, L. Determination of benzoic and sorbic acids in packaged vegetable products. Comp. Anal. 1995, 120, 2483-2487.

7. Wood, R.; Foster, L.; Damant, A.; Key, P. Analytical Methods for Food Additives; CRC Press: Boca Raton, FL, USA, 2000; pp. 54-72.

8. Shan, D.; Li, Q.; Xue, H.; Cosnier, S. A highly reversible and sensitive tyrosinase inhibition-based amperometric biosensor for benzoic acid monitoring. Sens. Actuators B 2008, 134, 1016-1021.

9. Kochana, J.; Kozak, J.; Skrobisz, A.; Woźniakiewicz, M. Tyrosinase biosensor for benzoic acid inhibition-based determination with the use of a flow-batch monosegmented sequential injection system. Talanta 2012, 96, 147-152.

10. Shan, D.; Shi, Q.; Zhu, D.; Xue, H. Inhibitive detection of benzoic acid using a novel phenols biosensor based on polyaniline-polyacrylonitrile composite matrix. Talanta 2007, 72, 1767-1772.

11. Li, S.; Tan, Y.; Wang, P.; Kan, J. Inhibition of benzoic acid on the polyaniline-polyphenol oxidase biosensor. Sens. Actuators B 2010, 144, 18-22.

12. Fontecave, M.; Pierre, J.-L. Oxidation by copper metalloenzymes and some biomimetic approaches. Coord. Chem. Rev. 1998, 170, 125-140.

13. Parvez, S.; Kang, M.; Chung, H.S.; Bae, H. Naturally occurring tyrosinase inhibitors: Mechanism and applications in skin health, cosmetics and agriculture industries. Phytother. Res. 2007, 21, 805-816.

14. Morales, M.D.; Morante, S.; Escarpa, A.; González, M.C.; Reviejo, A.J.; Pingarrón, J.M. Design of a composite amperometric enzyme electrode for the control of the benzoic acid content in food. Talanta 2002, 57, 1189-1198.

15. Narli, I.; Kiralp, S.; Toppare, L. Preventing inhibition of tyrosinase with modified electrodes. Anal. Chim. Acta 2006, 572, 25-31.

16. Lupetti, K.O.; Vieira, I.C.; Fatibello-Filho, O. Flow injection spectrophotometric determination of isoproterenol using an avocado (Persea americana) crude extract immobilized on controlled-pore silica reactor. Talanta 2002, 57, 135-143. 
17. Böyükbayram, A.E.; Kiralp, S.; Toppare, L.; Yagci, Y. Preparation of biosensors by immobilization of polyphenol oxidase in conducting copolymers and their use in determination of phenolic compounds in red wine. Bioelectrochemistry 2006, 69, 164-171.

18. Lupu, S.; Lete, C.; Balaure, P.C.; Caval, D.I.; Mihailciuc, C.; Lakard, B.; Hihn, J.-Y.; del Campo, F.J. Development of amperometric biosensors based on nanostructured tyrosinase-conducting polymer composite electrodes. Sensors 2013, 13, 6759-6774.

19. Rodrigues, V.C.; de Moraes, M.L.; Brisolari, A.; Soares, J.C.; Ferreira, M.; Gonçalves, D. Polypyrrole/phytase amperometric biosensors for the determination of phytic acid in standard solutions. Sens. Actuators B Chem. 2011, 160, 222-226.

20. Ghosh, S.; Graham, A.B.; Cooney, R.P.; Seakins, J.M. Infrared and Raman spectroscopic studies of the electrochemical oxidative degradation of polypyrrole. Synth. Met. 1998, 95, 63-67.

21. Tamm, J.; Marandi, J.M.; Tamm, T.; Tamm, L. Study of the properties of electrodeposited polypyrrole films. Russ. J. Electrochem. 2004, 40, 388-392.

22. Skompska, M.; Szkurlat, A. The influence of the structural defects and microscopic aggregation of poly(3-alkylthiophenes) on electrochemical and optical properties of the polymer films: Discussion of an origin of redox peaks in the voltammograms. Electrochim. Acta 2001, 46, 4007-4015.

23. Chang, T.S. An updated review of tyrosinase inhibitors. Int. J. Mol. Sci. 2009, 10, 2440.

24. Hazamh, H.H.; Yasof, N.A.; Salleh, A.B.; Bakar, F.A. An optical test strip for the detection of benzoic acid in food. Sensors 2011, 11, 7302-7313.

(C) 2014 by the authors; licensee MDPI, Basel, Switzerland. This article is an open access article distributed under the terms and conditions of the Creative Commons Attribution license (http://creativecommons.org/licenses/by/3.0/). 